\title{
Physisorbed CO on ionic crystals: An extended BEG spin-lattice model of adsorbed dipolar molecules
}

\author{
T.E. Burns and J.R. Dennison* \\ Department of Physics, Utah State University, Logan, UT 84322-4415
}

(Received

\begin{abstract}
The 2D dielectric phases and phase transitions of adsorbed dipolar molecules are studied using a dilute spin-one Ising model. The spin-one formulation assigns a spin $S_{i}= \pm 1$ to a (up/down) dipole occupying a lattice site $i$ adsorbed perpendicular to the substrate surface and $S_{i}=0$ to unoccupied sites. We relate the mean field expression for the spin-model interaction energy to a more detailed microscopic model involving dipolar, quadrupolar and dispersive interactions beyond nearest neighbor. Analytic solutions in the mean field approximation are discussed for dipole-orientation order-disorder transitions and ferroelectric-to-antiferroelectric transitions as a function of temperature and coverage. The model is applied to two prototypical dipolar physisorbed systems, $\mathrm{CO}$ on $\mathrm{MgO}(100)$ and $\mathrm{CO}$ on $\mathrm{NaCl}(100)$, using previous experimental and theoretical studies to determine the interaction energy parameters. We find, for most of these model parameters, that only temperature-dependent ferroelectric dipole order-disorder phase transitions can occur for these two systems. However, we find that phase transitions between the two ferroelectric states, driven by changes in coverage, should also be observed for some predicted values of the parameters. Phase diagrams are presented. Application of the model to other related adsorbed dipolar systems is also discussed.
\end{abstract}

Keywords: Surface thermodynamics; Ising models; Dipolar molecules; Carbon monoxide; Magnesium oxides; Sodium chloride.

PACS Numbers: 05.50.+q, 64.60.Cn, 64.60.-i, 68.35.Rh, 75.10.Hk, 75.70.Ak, 77.55.+f, 77.80.Bh

"Corresponding author: (801) 797-2936. FAX (801) 797-2492. E-mail PHYSJRD@CC.USU.EDU. 


\section{INTRODUCTION}

Monolayer adsorption of asymmetric molecules can lead to interesting observable phases and phase transitions which depend on adsorbate orientation. Such orientation-dependent interactions can result from steric, magnetic, electric, or chemical asymmetries of the adsorbates. Both the binding energy to the substrate and the interaction energy between admolecules can depend on adsorbate orientation. Dipolar adsorbates, such as CO, are a particularly interesting class of asymmetric adsorbates which have received considerable attention in recent years. ${ }^{1}$ In this paper, we develop a simple model for adsorption of dipolar molecules with two allowed dipole orientations as a spin-lattice system using a spin-1 Ising model in the mean field approximation.

Distinctive 2D dielectric (dipole ordering) phases and phase transitions can occur due to asymmetric dipolar interactions, as shown schematically for one hypothetical system in Fig. 1(a). If the adsorbate-substrate interaction has an energetically preferred orientation due to a strong substrate field, a zero-temperature ferroelectric configuration is favored, i.e., a phase where all adsorbed molecules align with the substrate field [FE phase in Fig. 1(a)]. A phase transition [at $\mathrm{T}_{\text {od }}$ ] may exist from a ferroelectric state to a disordered dipole (spin) orientation state [SDO phase] as $\mathrm{T}$ is increased. This is analogous to a paramagnetic system, where spins align with an applied magnetic field at low temperature, but entropy effects destroy this order at higher $\mathrm{T}^{2}$ The lowest interaction energy configuration for electric dipole pairs is with dipoles in opposition. Thus, strong adsorbate-adsorbate dipole interactions can induce a ground state configuration which is antiferroelectric or ferrielectric [AFE phase]. The dipole-dipole interaction energy becomes more important with decreasing separation or increasing coverage; therefore, a phase transition at low temperature from a FE phase to an AFE phase can occur [at $1_{C}$ ] driven by increasing coverage. A similar coverage-driven transition from one ferroelectric orientation to the other ferroelectric orientation is possible. The AFE phase may also exhibit a dipole-orientation order-disorder transition [at $\mathrm{TN}_{\mathrm{d}}$ ] driven by temperature.

This preliminary study has two primary objectives: (1) to develop a simple model that predicts, for the adsorption of dipoles on a square lattice with only two allowed orientations, which 2D dielectric phases will exist and the approximate range of transition temperatures; and (2) to test the results of our general model by application to prototypic experimental systems. We present the model and discuss possible phases and phase transitions for a general adsorption system in Section II. Section III describes our method of determining the interaction energies required for the model. The spin lattice model is then applied in Section IV to two specific, well-studied dipolar physisorption systems: $\mathrm{CO}$ on $\mathrm{MgO}(100)$ and $\mathrm{CO}$ on $\mathrm{NaCl}(100)$. We determine the energy parameters for the Ising model Hamiltonian from interaction energy calculations based on previous experimental and theoretical studies of the two systems. Possible phase diagrams for these two systems are presented. We also discuss the application of this model to other adsorbed dipole systems and present possible extensions of this work in Section V.

\section{SPIN-LATTICE MODEL}

The adsorption of dipolar molecules with only two allowed dipole orientations can be modeled as a 2D spin-lattice problem using a spin-1 Ising model. ${ }^{3}$ This model is equivalent to the symmeterized direct product of two spin- $1 / 2$ systems: ${ }^{4,5}$ a lattice-gas system modeling adsorption site occupation and a spin-lattice system modeling dipole orientation of occupied sites. For the $\mathrm{CO}$ on $\mathrm{MgO}$ and $\mathrm{NaCl}$ systems, we assign a spin $\mathrm{S}_{\mathrm{i}}=1\left[\mathrm{~S}_{\mathrm{i}}=-1\right]$ to a $\mathrm{CO}$ molecule adsorbed with the C-down [O-down] at a lattice site $\mathrm{i}$ and a spin $\mathrm{S}_{\mathrm{i}}=0$ to an empty lattice site $\mathrm{i}$. The spin-1 system, when limited to ferroelectric solutions, has two kinematically coupled order parameters: $<\mathrm{S}\rangle$ for the spin-lattice system and $\left\langle\mathrm{S}^{2}\right\rangle$ for the lattice-gas system. Antiferroelectric ordering is considered by splitting the lattice into two interpenetrating sublattices, labeled $\mathbf{u}$ and $\mathbf{d}{ }^{3,6}$ The antiferroelectric model has four order parameters: the average spins on the $\mathbf{u}$ and $\mathbf{d}$ sublattices $\left\langle S_{\left(\begin{array}{l}u \\ d\end{array}\right)}\right\rangle \equiv M_{\left(\begin{array}{l}u \\ d\end{array}\right)}$ and the average site occupations on the $\mathbf{u}$ and $\mathbf{d}$ sublattices

$\left\langle S_{\left(\begin{array}{l}u \\ d\end{array}\right)}^{2}\right\rangle \equiv \Theta_{\left(\begin{array}{l}u \\ d\end{array}\right)}$. Complete antiferroelectric ordering occurs when all spins on one sublattice are aligned and all spins on the other sublattice are antialigned, e.g. when $\left\langle\mathrm{S}_{\mathrm{u}}\right\rangle=+1$ and $\left\langle\mathrm{S}_{\mathrm{d}}\right\rangle=-1$.

Our model Hamiltonian for the full lattice, 


$$
\begin{aligned}
\mathscr{H}= & \mathscr{H}_{\text {int }}+\mathscr{H}_{\text {sub }} \\
= & \sum_{\langle i, j\rangle}\left[J S_{\mathrm{u}_{i}} S_{\mathrm{d}_{j}}+K S_{\mathrm{u}_{i}}^{2} S_{\mathrm{d}_{j}}^{2}+L\left(S_{\mathrm{u}_{i}} S_{\mathrm{d}_{j}}^{2}+S_{\mathrm{u}_{i}}^{2} S_{\mathrm{d}_{j}}\right)\right] \\
& -\sum_{i}\left[\Delta\left(S_{\mathrm{u}_{i}}^{2}+S_{\mathrm{d}_{i}}^{2}\right)+H\left(S_{\mathrm{u}_{i}}+S_{\mathrm{d}_{i}}\right)\right],
\end{aligned}
$$

is an extended Blume-Emery-Griffiths (BEG) ${ }^{7}$ model which includes symmetry breaking terms $\mathrm{L}$ and $\mathrm{H}$. This is the most general Hamiltonian possible for a spin-1 Ising system. ${ }^{4,8}$ For ferroelectric solutions, the $\mathbf{u}$ and $\mathbf{d}$ sublattices are equivalent and the subscripts may be dropped. $\mathscr{H}$ is the sum of a summation over nearest neighbors $<\mathrm{i}, \mathrm{j}\rangle$ of the interaction between the admolecules $\left(\mathscr{H}_{\text {int }}\right.$ ), and a summation over all lattice sites i of the adsorbate-substrate energies $\left(\mathcal{H}_{\text {sub }}\right)$. Both summations depend on the specific details of the spin orientations and use the notation

$\mathrm{J}=1 / 4\left(\mathrm{~J}_{\mathrm{uu}}+\mathrm{J}_{\mathrm{dd}}-2 \mathrm{~J}_{\mathrm{ud}}\right)$,

$\mathrm{K}=1 / 4\left(\mathrm{~J}_{\mathrm{uu}}+\mathrm{J}_{\mathrm{dd}}+2 \mathrm{~J}_{\mathrm{ud}}\right)$,

$\mathrm{L}=1 / 4\left(\mathrm{~J}_{\mathrm{uu}}-\mathrm{J}_{\mathrm{dd}}\right)$,

$\Delta=1 / 2\left(\mu_{\mathrm{u}}+\mu_{\mathrm{d}}\right)$, and

$\mathrm{H}=1 / 2\left(\mu_{\mathrm{u}}-\mu_{\mathrm{d}}\right)=(1 / 2 \beta) \ln \left\{\left[1+\exp \left(-\epsilon_{\mathrm{bd}} \beta\right)\right] /\left[1+\exp \left(-\epsilon_{\mathrm{bu}} \beta\right)\right]\right\}$,

where $\beta \equiv\left(k_{B} T\right)^{-1}, J_{u u}\left(J_{d d}\right)$ is the interaction energy between two neighboring molecules adsorbed spin-up (spin-down) [see Figs. 1(c) (i) and (iii)], $\mathrm{J}_{\mathrm{ud}}$ is the interaction energy between neighboring molecules adsorbed with spins in opposition [one spin-up and one spin-down, see Fig. 1(c) (ii)], $\mu_{\mathrm{u}}\left(\mu_{\mathrm{d}}\right)$ is the chemical potential of a molecule adsorbed spin-up (spin-down) [Fig. 1(b)], and $\epsilon_{\mathrm{bd}}>0\left(\epsilon_{\mathrm{bu}}>0\right)$ is the binding energy of a molecule adsorbed spin-up (spin-down).

It is the interplay of these energy parameters which determines the phase diagram of a particular system. Physically, J (bilinear term) is the interaction energy difference between a heterogeneous, phase separated ferroelectric state ( $\mathrm{M}=0$ with two domains, one all spin-up and one all spin-down) and a homogeneous antiferroelectric state $(M=0)$; $K$ (biquadratic term) is the average of the interaction energies; and $\mathrm{L}$ (crossed quadratic-linear term) is the difference in the two ferroelectric state $(\mathrm{M}= \pm 1)$ interaction energies. The constant $\Delta$ (substrate field strength) approaches the average binding energy at low temperature and $\mathrm{H}$ (substrate field asymmetry) approaches $1 / 2\left(\epsilon_{\mathrm{bu}}-\epsilon_{\mathrm{bd}}\right)$ at low T. In the mean field approximation, the interaction energy of any individual admolecule depends on the average dipole orientation and lattice occupation. The energy per site from Eq. (1) in the mean field approximation is ${ }^{9}$

$$
\begin{aligned}
& \frac{\left\langle\mathscr{H}\left(M_{\mathrm{u}}, M_{\mathrm{d}} \Theta_{\mathrm{u}}, \Theta_{\mathrm{d}}\right)\right\rangle}{N} \\
& =\frac{1}{2}\left[z J M_{\mathrm{u}} M_{\mathrm{d}}+z K \Theta_{\mathrm{u}} \Theta_{\mathrm{d}}+z L\left(M_{\mathrm{u}} \Theta_{\mathrm{d}}+M_{\mathrm{d}} \Theta_{\mathrm{u}}\right)\right. \\
& \left.\quad+\Delta\left(\Theta_{\mathrm{u}}+\Theta_{\mathrm{d}}\right)+H\left(M_{\mathrm{u}}+M_{\mathrm{d}}\right)\right]
\end{aligned}
$$

where $\mathrm{N}$ is the total number of lattice sites and $\mathrm{z}$ is the lattice coordination number ( $\mathrm{z}=4$ for a $2 \mathrm{D}$ square lattice).

The expectation value of the energy can be found by minimizing the Hamiltonian in Eq. (1) using a density matrix formalism. General analytic expressions, in the mean field approximation, for the average dipole orientations (spin) $M_{\left(\begin{array}{l}u \\ d\end{array}\right)}$, the coverages $\Theta_{\left(\begin{array}{l}u \\ d\end{array}\right)}$, and the ratios $M_{\left(\begin{array}{l}u \\ d\end{array}\right)} / \Theta_{(}\left(\begin{array}{l}u \\ d\end{array}\right)$ are: ${ }^{4}$ 


$$
\begin{aligned}
& 2 \exp \left[\beta\left(L z M_{(\mathrm{d})}+K z \Theta_{(\mathrm{d})}+\Delta\right)\right]
\end{aligned}
$$

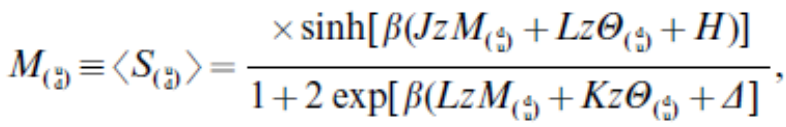

$$
\begin{aligned}
& \times \cosh \left[\beta\left(J z M_{(む)}+L z \Theta_{(\mathrm{d})}+H\right)\right]
\end{aligned}
$$

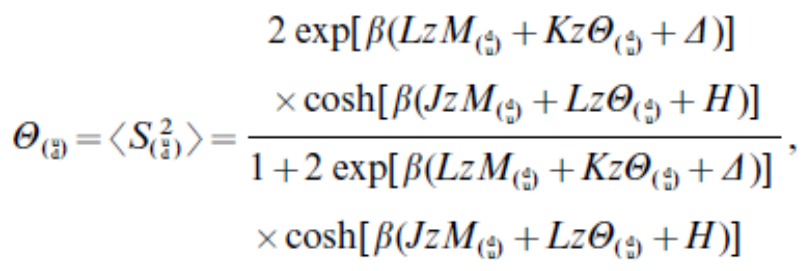

$$
\begin{aligned}
& \frac{M_{(\unlhd)}}{\Theta_{(घ)}}=\tanh \left[\beta\left(J z M_{(y)}+L z \Theta_{(\unlhd)}+H\right)\right] .
\end{aligned}
$$

The thermodynamics follow from the expressions ${ }^{4}$ for the equilibrium free energy $\phi_{E}=\beta^{-1} \ln \left[\left(1-\Theta_{u}\right)\left(1-\Theta_{d}\right)\right]+z\left[J M_{u} M_{d}\right.$ $\left.+\mathrm{L}\left(\mathrm{M}_{\mathrm{u}} \Theta_{\mathrm{d}}+\mathrm{M}_{\mathrm{d}} \Theta_{\mathrm{u}}\right)+\mathrm{K} \Theta_{\mathrm{u}} \Theta_{\mathrm{d}}\right]$ and the pressure $\mathrm{p}=-\phi_{\mathrm{E}}$.

The Hamiltonian in Eq. (1) has been studied in the mean field approximation for several different combinations of these energy parameters, in the absence of the asymmetric terms $\mathrm{L}$ and $\mathrm{H}^{6,7,10,11}$ Blume et al ${ }^{7}$ solved the case where $\mathrm{L}=\mathrm{H}=0$ and $\mathrm{J}>0$. Their results were applied to the phase separation of ${ }^{3} \mathrm{He}$ and ${ }^{4} \mathrm{He}$ and to the phases of a microemulsion. ${ }^{7,10}$ Lajzerowicz et al. studied cases where $\mathrm{J}=\mathrm{L}=\mathrm{H}=0$, and where $\mathrm{L}=\mathrm{H}=0$ with $\mathrm{J}>0$; the latter case was successfully applied to the condensation and solidification of a simple fluid. ${ }^{11}$ Hoston et al. ${ }^{6}$ studied this Hamiltonian for $\mathrm{J}<0$ and $\mathrm{K}<0$ with $\mathrm{L}=\mathrm{H}=0$ and considered antiferromagnetic ordering. Wu investigated the case where $\mathrm{J}=\mathrm{L}=0{ }^{5} \quad$ The full Hamiltonian in Eq. (1) (with $\mathrm{J}, \mathrm{K}, \mathrm{L}, \Delta$ and $\mathrm{H} \neq 0$ and $\mathrm{J}>0$ ) has also been studied in the mean field approximation by Sivardiere et al. ${ }^{4}$ However, they used numerical calculations to determine the very complicated phase diagrams for only $\mathrm{K}>0$ with $\mathrm{L}=0.5$. Sivardiere et al. did not consider antiferromagnetic ordering.

None of the above studies considered energy parameters which fully describe the adsorption systems examined here. For our applications, where $\mu_{\mathrm{u}}, \mu_{\mathrm{d}}>0, \Delta$ is always positive and the $S=+1$ state can always be chosen so that $\mathrm{H}$ is nonnegative. However, since $\mathrm{J}_{\mathrm{uu}}, \mathrm{J}_{\mathrm{dd}}$, and $\mathrm{J}_{\mathrm{ud}}$ each can in general be either attractive or repulsive, $\mathrm{J}$, $\mathrm{K}$, and $\mathrm{L}$ may take on any combination of signs. To illustrate the interplay of the energy parameters, consider the possible ground states at zero temperature. For dipolar adsorption, $\mathrm{L}=0$ would imply there is no interaction energy asymmetry (e.g., no induced dipoles), while $\mathrm{H}=0$ would imply there is no orientational dependence in the binding energy. When $\mathrm{H}>>|\mathrm{J}|,|\mathrm{L}|$, binding energy asymmetries are dominant and only a ferroelectric ground state occurs. However, in the regime where $\mathrm{H}<|\mathrm{J}|$ or $\mathrm{H}<|\mathrm{L}|$, more phases are possible. If the interaction energies for the three possible dipole configuration pairs in Fig. 1(c) are equal ( $\mathrm{J}_{\mathrm{ud}}=\mathrm{J}_{\mathrm{dd}}=\mathrm{J}_{\mathrm{uu}}$ ), then $\mathrm{J}=0$ and $\mathrm{L}=0$; here the standard results of Yang and Lee ${ }^{12}$ are immediately recoverable. In this case, only the binding interactions depend on alignment. Further, in the limit $\mathrm{H} \rightarrow 0_{+}$with $\mathrm{J}=\mathrm{L}=0$, , becomes symmetric and no dipole ordering is expected. If $\mathrm{J}>0\left(\mathrm{~J}_{\mathrm{uu}}+\mathrm{J}_{\mathrm{dd}}>\right.$ $\left.2 J_{u d}\right)$, a ferroelectric state is expected when one spin orientation is energetically preferred over another $(\mathrm{L} \neq 0$ or $\mathrm{H} \neq 0)$. Antiferroelectric or ferrielectric states require $\mathrm{J}<0\left(2 \mathrm{~J}_{\mathrm{ud}}>\mathrm{J}_{\mathrm{uu}}+\mathrm{J}_{\mathrm{dd}}\right)$. This implies that the interaction energy between two opposed dipoles [see Fig. 1(c) ii)] is less than the average interaction energy between two aligned dipoles with either orientation [Figs. 1(c) i) and iii)].

In Section IV we show for our two example systems, $\mathrm{CO}$ on $\mathrm{NaCl}$ and $\mathrm{CO}$ on $\mathrm{MgO}$, that $\Delta » 0$ and $\mathrm{H}>0$ and that $\mathrm{J}<0, \mathrm{~K}<0$, and $\mathrm{L}<0$ since $0>\mathrm{J}_{\mathrm{dd}}>\mathrm{J}_{\mathrm{ud}}>\mathrm{J}_{\mathrm{uu}}$ and $\mathrm{J}_{\mathrm{ud}}>1 / 2\left(\mathrm{~J}_{\mathrm{uu}}+\mathrm{J}_{\mathrm{dd}}\right)$. We also show that only FE ordering can be expected for these systems. All the results of this section also apply to purely FE systems. Antiferroelectric ordering is considered in subsequent sections only where explicitly noted. Specific applications of the antiferroelectric equations to the adsorption of dipolar molecules are found elsewhere. ${ }^{13}$ 


\section{INTERACTION POTENTIAL CALCULATIONS}

We now apply the model specifically to $\mathrm{CO}$ physisorbed on $\mathrm{MgO}(100)$ and $\mathrm{NaCl}(100)$ surfaces; in these systems a dipole adsorbed C-down (O-down) has $\mathrm{S}=+1(-1)$ and an empty site has $\mathrm{S}=0 . \quad \mathrm{J}_{\mathrm{uu}} \rightarrow \mathrm{J}_{\mathrm{cc}}\left(\mathrm{J}_{\mathrm{dd}} \rightarrow \mathrm{J}_{\mathrm{oo}}\right)$ represents the interaction between two C-down (O-down) dipoles and $\mathrm{J}_{\mathrm{ud}} \rightarrow \mathrm{J}_{\mathrm{co}}$ represents the interaction between dipoles in opposition (see Fig. 1). Equation (2) follows with the appropriate subscript substitutions.

To apply our spin-lattice model, the interaction energy parameters J, $\mathrm{K}$ and $\mathrm{L}$ must be evaluated in the mean field approximation. We relate the mean field expression for the spin lattice model average interaction energy per lattice site $<\mathscr{H}_{\text {int }}>/ \mathrm{N}$ from Eq. (3) to a more detailed microscopic potential $<\mathrm{U}_{\text {int }}>$ described below. These two mean field approaches yield identical results when interactions between occupied sites are distance-independent. This is the case for three important types of interactions: (1) where interactions are very short range and only first nearest neighbor interactions are non-negligible, (2) for infinite range interactions where the interactions are the same for all sites, and (3) when all interaction energies are negligible. ${ }^{14}$ However, the interactions involved in our problem are long-range and distance-dependent. We show in this section how to accommodate this complication for a number of specific cases.

The microscopic interaction potential at site $\mathrm{i}$ of $\mathrm{N}$ sites is $\mathrm{U}_{\text {int }}^{\mathrm{i}}=\mathrm{U}_{\text {dipole }}^{\mathrm{i}}+\mathrm{U}_{\text {quad }}^{\mathrm{i}}+\mathrm{U}_{\text {disp }}^{\mathrm{i}}$. The dipole-dipole interaction, $U_{\text {dipole }}$, is the interaction energy for an infinite 2D lattice of classical electric dipoles having spins $S_{i}$ and $S_{j}$ with separations $r_{i j}$ and dipole moments $p_{i}$ and $p_{j}$ [equal to $p_{u}$ or $p_{d}$ (see Fig. 1)]. The quadrupole-quadrupole interaction energy, $U_{\text {quad }}$, for an infinite 2D lattice of classical quadrupoles with quadrupole moments $q_{i}$ and $q_{j}$ is independent of the orientation of each linear quadrupole restricted to parallel orientation (and hence depends only on site occupation or $\mathrm{S}_{\mathrm{i}}{ }^{2}$ ). The dipole-quadrupole interaction is zero for a linear quadrupole aligned parallel to a dipole. $\mathrm{U}_{\text {dipole }}$ and $\mathrm{U}_{\text {quad }}$ were calculated as pair-wise sums of classical point dipoles and linear quadrupoles. ${ }^{9,15}$ Our calculations of the dispersion (hard-core repulsion) interaction, $\mathrm{U}_{\text {disp, }}$, used a distance-dependent, orientation-independent exponential form determined by Ben-Ephriam et al., ${ }^{16}$ which we limited to only nearest neighbor interactions. ${ }^{15}$

The microscopic interaction potential can be written in a terms of spin notation ${ }^{17}$ as:

$$
\begin{aligned}
U_{\mathrm{int}}^{i}= & \frac{1}{32 \pi \epsilon_{0}} \sum_{j \neq i}\left[\frac{\left(p_{\mathrm{C}}-p_{\mathrm{o}}\right)^{2}}{r_{i j}^{3}} S_{i} S_{j}+\frac{\left(p_{\mathrm{C}}+p_{\mathrm{O}}\right)^{2}}{r_{i j}^{3}}\right. \\
& \left.\times S_{i}^{2} S_{j}^{2}+\frac{\left(p_{\mathrm{C}}^{2}-p_{\mathrm{O}}^{2}\right)}{r_{i j}^{3}}\left(S_{i} S_{j}^{2}+S_{i}^{2} S_{j}\right)\right] \\
& +\frac{1}{8 \pi \epsilon_{0}} \sum_{j \neq i} \frac{q_{i} q_{j}}{r_{i j}^{5}} S_{i}^{2} S_{j}^{2}+\frac{1}{2} \sum_{j \neq i} B S_{i}^{2} S_{j}^{2} \mathrm{e}^{-c r_{i j}} .
\end{aligned}
$$

In general, evaluation of $U_{\text {int }}$ (and hence determination of $\mathrm{J}, \mathrm{K}$, and $\mathrm{L}$ ) requires knowledge of the specific dipole occupation $S_{\mathrm{i}}$ and orientation $\mathrm{p}_{\mathrm{i}}$ on each lattice site. However, we can apply the mean field approximation to Eq. (5) and evaluate the result, $\left\langle\mathrm{U}_{\text {int }}\right\rangle$, at specific coverages for the three dipole configurations shown in Fig. 1(c). We then equate these to the corresponding values of $\left\langle\mathscr{H}_{\text {int }}\right\rangle / \mathrm{N}$ in Eq. (3). From Eq. (3), in the mean field approximation, we have 


$$
\begin{gathered}
\left\langle\mathscr{H}_{\text {int }}^{+}(M, \Theta)\right\rangle / N=\frac{-z \Theta^{2}}{2}(J+2 L+K) \\
=\frac{-z \Theta^{2}}{2} J_{\mathrm{CC}}, \quad M=+\Theta, \\
\left\langle\mathscr{H}_{\text {int }}^{0}(M, \Theta)\right\rangle / N+\frac{-z \Theta^{2}}{2}(K) \\
=\frac{-z \Theta^{2}}{8}\left(J_{\mathrm{CC}}+J_{\mathrm{OO}}+2 J_{\mathrm{CO}}\right), \quad M=0, \\
\left\langle\mathscr{H}_{\text {int }}^{-}(M, \Theta)\right\rangle / N=\frac{-z \Theta^{2}}{2}(J-2 L+K) \\
=\frac{-z \Theta^{2}}{2} J_{\mathrm{OO}}, \quad M=-\Theta,
\end{gathered}
$$

where the superscripts +, 0 , and - denote C-down FE, disordered or AFE, and O-down FE configurations, respectively. Note Eq. (6) only depends on $\mathrm{M}$ and $\Theta$ and is independent of the specific ordering of the dipoles on the lattice, while Eq. (5) is not independent of ordering. Application of the mean field approximation to Eq. (5), for the same values of $\mathrm{M}$ as in Eq. (6) and at full coverage $e^{18} \Theta=1$, yields a result independent of ordering:

$$
\begin{aligned}
\left(\begin{array}{c}
\left.\left\langle{ }^{0} U_{\text {int }}^{+} \Theta, \Theta=1\right)\right\rangle \\
\left\langle{ }^{0} U_{\text {int }}^{0}(M=0, \Theta=1)\right\rangle \\
\left\langle U_{\text {int }}^{-}(M=-\Theta, \Theta=1)\right\rangle
\end{array}\right) \\
\equiv\left(\begin{array}{c}
\left\langle{ }^{\circ} U_{\text {dipole }}^{+}\right\rangle \\
\left\langle{ }^{0} U_{\text {dipole }}^{0}\right\rangle \\
\left\langle{ }^{0} U_{\text {dipole }}^{-}\right\rangle
\end{array}\right)+U_{\text {quad }}^{0}+U_{\text {rep }}^{0} \\
=\left(\begin{array}{c}
p_{\mathrm{C}}^{2} \\
\frac{1}{4}\left(p_{\mathrm{C}}+p_{\mathrm{O}}\right)^{2} \\
4 \pi \epsilon_{0} \\
\sum_{i=1}^{N} r_{i}^{-3} \\
p_{\mathrm{O}}^{2}
\end{array}\right) \frac{1}{q_{2}} \sum^{N} r_{i}^{-5}+\frac{z B}{2 \pi \epsilon_{0}} \mathrm{e}^{-c r_{\mathrm{nn}}},
\end{aligned}
$$

where the superscript ${ }^{\circ}$ indicates values at full coverage, and $r_{n n}$ is the first nearest-neighbor distance. Equating $<^{0} \mathrm{U}_{\text {int }}(\Theta=1)>/ \mathrm{N}$ from Eq. (7) with $<\mathrm{H}_{\text {int }}>/ \mathrm{N}$ from Eq. (6) thus uniquely determines the three mean field interaction energy parameters $\mathrm{J}, \mathrm{K}$, and $\mathrm{L}$ (or $\mathrm{J}_{\mathrm{cc}}, \mathrm{J}_{\mathrm{oo}}$, and $\mathrm{J}_{\mathrm{oc}}$ ) at full coverage. From Eqs. (6) and (7), it can readily be shown that $\mathrm{J}<0$ (which implies there can be no phase separation) and that $\mathrm{K}<0$. Likewise, $\mathrm{L}>0$ if $\left|\mathrm{p}_{\mathrm{o}} / \mathrm{p}_{\mathrm{c}}\right|>1$ and $\mathrm{L}<0$ otherwise.

At less than full coverage, lattice site occupation must be specified in even more detail to evaluate $\left\langle U_{\text {int }}(\Theta)>\right.$, since $S_{i}$ can be 0 (in addition to \pm 1 ); one cannot evaluate the summations in Eq. (5) without complete knowledge of which $r_{i}$ have $S_{i}=0$. Therefore, we consider three limiting cases for the strength of the interaction energies which lead to different growth modes and to well-determined ordering in the spin-lattice systems.

Case I: At less than full coverage strong, attractive adsorbate interactions cause the admolecules to form islands, or clusters, of full coverage structure with regions of unoccupied sites. For extreme interactions, a single island will form. In the thermodynamic limit $(\mathrm{N} \rightarrow \infty)$, where edge or finite-size effects are negligible, $<\mathrm{U}_{\text {int }}(\mathrm{M}, \Theta)>\rightarrow \Theta<{ }^{0} \mathrm{U}_{\text {int }}(\mathrm{M})>$, which scales as the fractional area occupied by the full coverage raft. Equating this with Eq. (6), we see that the energy parameters J, K, and L are proportional to $\Theta^{-1}$ for Case I.

Case II: When interaction energies are distance independent, site occupation is entirely random. In the limit of weak interaction energies and for infinite range interactions, there also is no correlation between occupancy of 
adjacent sites. Here, each term in the summations of Eq. (7) is multiplied by the probability that both sites are occupied, $\Theta^{2}$, and $<\mathrm{U}_{\text {int }}(\mathrm{M}, \Theta)>\rightarrow \Theta^{2}<{ }^{0} \mathrm{U}_{\text {int }}(\mathrm{M})>$. This yields coverage independent expressions for $\mathrm{J}, \mathrm{K}$, and $\mathrm{L}$, as is to be expected in Case II; the mean field approximation is exact. ${ }^{14}$

Case III: The case for strong, repulsive adsorbate interactions is more complicated. Adsorbates prefer to form dilute phases with the admolecules as far apart as possible. $<\mathrm{U}_{\text {int }}(\mathrm{M}, \Theta)>$ is readily determined at coverages where square superlattice structures form. For such superlattices, the distances in Eq. (7) can be rescaled to the full coverage separations $r_{i j}(\Theta)=\Theta^{-1 / 2} r_{i j}{ }^{0}$. For Case III, Eq. (7) becomes

$$
\begin{aligned}
\left(\begin{array}{c}
\left\langle U_{\text {int }}^{+}(M, \Theta)\right\rangle \\
\left\langle U_{\text {int }}^{0}(M, \Theta)\right\rangle \\
\left\langle U_{\text {int }}^{-}(M, \Theta)\right\rangle
\end{array}\right)= & \Theta^{3 / 2}\left(\begin{array}{c}
{ }^{\circ} U_{\text {dipole }}^{+} \\
{ }^{\circ} \\
{ }_{\text {dipole }}^{0} \\
U_{\text {dipole }}^{-}
\end{array}\right) \\
& +\Theta^{5 / 2} U_{\text {quad }}^{0}+(z B) \mathrm{e}^{-c \Theta^{-1 / 2} r_{n n}^{0}}
\end{aligned}
$$

Finally, for strong repulsive interactions, which favor growth of dilute phases, we can equate Eqs. (8) and (6) and solve for the interaction energy parameters as a function of coverage:

$$
\begin{aligned}
J(\Theta)= & -\frac{\Theta^{-1 / 2}}{z}\left({ }^{\circ} U_{\text {dipole }}^{+}+{ }^{\circ} U_{\text {dipole }}^{-}-2{ }^{\circ} U_{\text {dipole }}^{0}\right), \\
K(\Theta)= & \frac{-2 \Theta^{-1 / 2}}{z}\left[U_{\text {dipole }}^{0}+\Theta U_{\text {quad }}^{0}\right. \\
& \left.+\Theta^{-3 / 2}(z B) \mathrm{e}^{-c \Theta^{-1 / 2} r^{\theta n}}\right], \\
L(\Theta)= & -\frac{\Theta^{-1 / 2}}{2 z}\left({ }^{\circ} U_{\text {dipole }}^{+}-{ }^{\circ} U_{\text {dipole }}^{-}\right) .
\end{aligned}
$$

We expect Case III to describe $\mathrm{CO}$ on $\mathrm{NaCl}$ and $\mathrm{MgO}$, as discussed in the next section.

\section{APPLICATION TO CO ON NaCl (100) AND CO ON MgO (100)}

We now turn our attention to two specific systems with the simple dipolar adsorbate CO physisorbed to $\mathrm{NaCl}(100)$ and $\mathrm{MgO}(100)$ square-symmetry substrates. To apply our model and determine the possible 2D dielectric phases and phase transitions for the two systems, we calculate the microscopic potential, determine the predominant growth mode for each system, and evaluate the energy parameters in Eq. (2). We also verify that our simple model is applicable to $\mathrm{CO}$ on $\mathrm{NaCl}$ and $\mathrm{MgO}$.

Carbon monoxide is an asymmetric, diatomic molecule with a weak permanent dipole moment, ${ }^{19} \mathrm{p}_{\text {nat }}$ (see Table 1), and a strong quadrupole moment $\left(q=8.34 \times 10^{-40} \mathrm{C}-\mathrm{m}^{2}\right){ }^{20}$ The total electric dipole moment of adsorbed CO, $\mathrm{p}_{\text {tot }}$, equals $\mathrm{p}_{\text {nat }}+\mathrm{p}_{\text {ind }}$, where $\mathrm{p}_{\text {ind }}$ is the dipole moment induced in an adsorbed CO molecule by the substrate surface electric field. The interaction energy between $\mathrm{CO}$ admolecules, the total dipole moment, and the binding energies of $\mathrm{CO}$ to the substrate all depend on dipole orientation. ${ }^{21,22}$ Both $\mathrm{NaCl}$ and $\mathrm{MgO}$ have a rock salt structure, with lattice constants $\mathrm{a}=5.64 \AA$, and $\mathrm{a}=4.21 \AA$, respectively.

\section{A. Determination of Substrate Field Strength Parameters}

The adsorbate-substrate field strength parameters $\Delta$ and $\mathrm{H}$ are determined directly from binding energy calculations. As expected, the binding energy is dependent on adsorbate orientation and on adsorbate position due to 
substrate potential corrugation. To fulfill the model requirement of only two allowed energy states, we limit our application to vertical adsorption above cation sites. This restricts our study to commensurate, square-symmetry superlattices. This restriction is consistent, for the most part, with limited available data of the structure of the two systems, as discussed below.

$\mathrm{CO}$ adsorbs to the $\mathrm{NaCl}(100)$ surface with the $\mathrm{CO}$ bond axis perpendicular to the surface. ${ }^{23,24}$ The heat of adsorption has been measured. ${ }^{23,25,26} \mathrm{~A} 1 \mathrm{x} 1$ commensurate structure with perpendicular $\mathrm{CO}$ orientation has been observed with polarization ir spectroscopy, ${ }^{23,27,28}$ although at $\mathrm{T}<35 \mathrm{~K}$ for higher coverage a tilted $2 \mathrm{x} 1$ structure has been reported. ${ }^{29,30}$ Several theoretical studies have predicted that adsorption above $\mathrm{Na}^{+}$lattice sites is perpendicular, ${ }^{25,26,31-33}$ and have determined different binding energies for the C-down and O-down orientations. ${ }^{21,25} \mathrm{~A}$ recent calculation suggests that tilted structures may also be possible. ${ }^{34}$ For the $\mathrm{CO}$ on $\mathrm{NaCl}$ interactions in this study, we use binding energies for both perpendicular orientations calculated by Gready et al. (see Table 2). ${ }^{21}$

Theoretical studies of $\mathrm{CO}$ adsorbed on $\mathrm{MgO}(100)$ at lower coverages $(\Theta \leq 1 / 2 \mathrm{ML})$ have shown that the most energetically favored position for adsorption is above a $\mathrm{Mg}^{++}$site and that the $\mathrm{CO}$ bond axis is perpendicular to the surface at these sites. ${ }^{35-38}$ Positions above the $\mathrm{O}^{--}$sites and the $\mathrm{Mg}:: \mathrm{O}$ bonds are energetically disallowed. ${ }^{36-38}$ Theoretical studies have also determined binding energies which depend on vertical orientation. ${ }^{22,35,36,39,40}$ A wide range of binding energies for $\mathrm{CO}$ on $\mathrm{MgO}$ are reported in the literature. ${ }^{38}$ We apply our model to a range of differences in binding energies for the two orientations (Table 3), based on extremes in the listed binding energies (Table 2).

At higher coverages $(\Theta>1 / 2 \mathrm{ML})$ theoretical results ${ }^{38}$ show that significant tilting, including structures with CO molecules parallel to the surface, are energetically favored, due to adsorbate-adsorbate interactions. At $\Theta=1 / 2$ ML, Minote et al. find a structure with CO tilted along Mg-Mg bonds is slightly preferred, although other calculations predict $\mathrm{CO}$ is adsorbed perpendicularly above the Mg sites at $\Theta=1 / 2$ ML. $^{38}$ LEED studies found a series of uniaxially-compressed, higher-order commensurate structures with increasing coverages $\Theta>1 / 2$ ML as T decreased. ${ }^{41}$ Some CO molecule tilting is inferred in these LEED studies. These results are consistent with theoretical studies which determined that the Mg troughs along the $[1,1,0]$ direction are essentially potential energy minima for CO adsorption. ${ }^{37}$ High resolution polarization ir ${ }^{42}$ and He atom scattering ${ }^{43}$ studies have observed a transition, as $\mathrm{T}$ is decreased below $45 \mathrm{~K}$, from a square symmetry phase with $\mathrm{CO}$ adsorbed C-down above Mg sites to a more complex structure with tilted CO molecules; these are observed at coverages $\$ 1 / 2 \mathrm{ML}$. At lower coverages, only perpendicular adsorption is observed. ${ }^{42}$ These higher coverage studies are inconsistent with our simple model restricted to adsorption over cation sites. However, our analysis of the adsorbate-adsorbate interaction potential in Sec. III.B limits the applicable region of our model to $\Theta \$ 1 / 2$ ML, that is to coverages less than those in these studies.

\section{B. Calculation of Adsorbate Interaction Energy Parameters}

${ }^{\circ} U_{\text {dipole }}$ was evaluated for the three dipole orientation configurations shown in Fig. 1(c); values are listed in Table 3(a). To calculate ${ }^{\circ} U_{\text {dipole }}$, $p_{\text {ind }}$ and $p_{\text {nat }}$ must be known (Table 1). The orientationally-dependent induced dipole moments for $\mathrm{CO}$ on $\mathrm{NaCl}$ were determined from calculations of the polarization contribution to the total binding energy of the $\mathrm{CO}$ molecule. ${ }^{21}$ Pacchioni et al. calculated induced dipole moments for both vertical orientations of $\mathrm{CO}$ on $\mathrm{MgO}^{22}$ For both substrates and both orientations, $\mathrm{p}_{\text {ind }}>\mathrm{p}_{\text {nat }}$; therefore, the total dipole moment always points away from the crystal surface [see Fig. 1(c)]. Also, we note that $\mathrm{p}_{\text {tot }}$ for the C-down orientation is larger than $\mathrm{P}_{\mathrm{tot}}$ for the O-down orientation for both substrates. This implies repulsive adsorbate interactions (Case III) such that $0>\mathrm{J}_{\mathrm{oo}}>\mathrm{J}_{\mathrm{co}}>\mathrm{J}_{\mathrm{cc}}$ for all orientations of the admolecules, and that an antiferroelectric or ferrielectric state is not energetically favored.

The repulsive nature of the CO-CO interactions favor dilute adsorption instead of random adsorption or island growth of denser phases. Therefore, theoretically we expect Eq. (9) developed for strongly repulsive interactions (Case III) to be most applicable. However, the growth mode in the submonolayer region for $\mathrm{CO}$ on $\mathrm{NaCl}$ has been inferred from ir spectroscopy to follow the Langmuir model, with random adsorbate distribution over the substrate lattice (Case II). ${ }^{26}$ A similar growth mode would be expected for $\mathrm{CO}$ on MgO, but has not yet been measured. Because of the uncertainty in growth mode, values of the spin model energy parameters as a function of coverage were calculated for all three growth modes and are shown in Fig. 2. $\quad<\mathrm{U}_{\mathrm{int}}>$ was evaluated at coverages $(1 \leq \Theta \leq 0.025 \mathrm{ML})$ corresponding to the 20 densest square superlattice structures.

$\mathrm{U}_{\text {disp }}$ determines primarily the minimum spacing possible between adsorbed $\mathrm{CO}$ molecules, and therefore places an upper limit on the coverage. The dispersion force constants used for $\mathrm{CO}$ are $\mathrm{B}=3.16 \mathrm{Gcal}^{-\mathrm{mol}^{-1}}$ and $\mathrm{c}=4.318 \AA^{-1}{ }^{16}$ Quadrupole-quadrupole interactions tend to tilt the molecules, which can introduce more than two orientational states and invalidate application of our model. $\mathrm{U}_{\text {quad }}$ has a shorter range than $\mathrm{U}_{\text {dipole }}$ and becomes more significant at higher coverages where admolecule separation is reduced.

For $\mathrm{CO}$ on $\mathrm{MgO}(100)$, $\mathrm{U}_{\text {disp }}$ is much greater than the binding energy for all three orientations at $\Theta=1 \mathrm{ML}$. 
Therefore, commensurate superlattices with coverages greater than $\Theta=1 / 2 \operatorname{ML}(\sqrt{2} x \sqrt{2}$ structure) are energetically unstable. $U_{\text {dipole }}$ is greater than both $U_{\text {quad }}$ and $U_{\text {disp }}$ for superlattice coverages of $\Theta \lesssim 1 / 2$ ML. Therefore, we conclude that our model is applicable to fully-occupied, square-symmetry, commensurate CO superlattice structures on $\mathrm{MgO}(100)$ where $\Theta \lesssim 1 / 2 \mathrm{ML}$ and where admolecules sit vertically above cations. This is consistent with experimental ${ }^{42,43}$ and theoretical ${ }^{38}$ results for $\Theta \leq 1 / 2 \mathrm{ML}$, as discussed above.

Similar conclusions are drawn for $\mathrm{CO}$ on $\mathrm{NaCl}(100)$. For this system: (1) $\mathrm{U}_{\text {disp }}$ is much less than the binding energy for all coverages up to $\Theta=1 \mathrm{ML}$; (2) $\mathrm{U}_{\text {disp }} \approx \mathrm{U}_{\text {dipole }}$ for $\Theta=1 \mathrm{ML}$ and $\mathrm{U}_{\text {disp }}$ « $\mathrm{U}_{\text {dipole }}$ at lower coverages; and (3) $\mathrm{U}_{\text {quad }} / \mathrm{U}_{\text {dipole }}$ for $\Theta>0.6 \mathrm{ML}$. We conclude that our model is applicable to all fully-occupied, square-symmetry, commensurate $\mathrm{CO}$ monolayer structures on $\mathrm{NaCl}(100)$ where admolecules sit above cations, although some quadrupole-induced tilting of the $\mathrm{CO}$ molecules may occur for coverages above $0.6 \mathrm{ML}$. Note, however, that theoretical studies ${ }^{21,25,33}$ that calculate the binding energy of $\mathrm{CO}$ on $\mathrm{NaCl}$ for different orientations above the cation have shown the perpendicular orientation to be by far the most energetically favored. The binding energies (Table 1) are one to two orders of magnitude larger than interaction energies (Table 3). Therefore, we conclude that any small increase in interaction energy due to $\mathrm{CO}$ tilting would be offset by a decrease in the binding energy, and that the molecules will not tilt significantly. For the $\mathrm{CO}$ on $\mathrm{NaCl}$ system, perpendicular adsorption has been observed (see discussion above), ${ }^{23,24}$ which is consistent with our analysis.

\section{Observable Changes in Dipole Orientation}

By solving Eq. 4(c) for $\mathrm{M}$ as a function of temperature at a variety of coverages, we can probe the thermodynamics and phase transitions of these systems. For $\mathrm{CO}$ on $\mathrm{NaCl}$, the magnitudes of the binding energy asymmetry $\mathrm{H}$ is much greater than the adsorbate-adsorbate interaction energy parameters $|\mathrm{J}|$ and $|\mathrm{L}|$. Therefore, one ferroelectric phase is preferred, there is still a residual net dipole orientation even at very high temperatures, and there will be no phase transitions. The results of our mean field approximation analysis for the spin-lattice model of CO on $\mathrm{NaCl}$ for all three Cases, using the parameter values in Table 3, are shown in Fig. 3(a). The low temperature configuration is a ferroelectric state with all molecules adsorbed C-down. Observable change in dipole orientation takes place near $1000 \mathrm{~K}$, well above both the desorption temperature $\left(\mathrm{T}_{\text {desorb }}=163 \mathrm{~K}\right)^{21}$ and the $2 \mathrm{D}$ melting temperature $\left(\mathrm{T}_{\mathrm{m}}=53 \mathrm{~K}\right){ }^{24}$ Changes in dipole orientation clearly will not be observable.

Similar results for $\mathrm{CO}$ on $\mathrm{MgO}$ are presented in Figs. 3(b), based on the upper limit of the binding energy differences $(\mathrm{H}=58.6 \mathrm{meV}$; see Table 1$)$ for all three cases. The low temperature configuration is a ferroelectric state with all C-down and observable change in dipole ordering occurs well above the $2 \mathrm{D}$ melting temperature $\left(\mathrm{T}_{\mathrm{m}}=55\right.$ $\mathrm{K})^{44}$ and the desorption temperature $\left(\mathrm{T}_{\text {desorb }}=180 \mathrm{~K}\right){ }^{44}$ Again, this will not be observable.

The results for $\mathrm{CO}$ on $\mathrm{MgO}$, using the lower limit in binding energy differences $(\mathrm{H}=5.2 \mathrm{meV})$, are shown in Fig. 3(c). For lower values of $H$, there is a substantial decrease in $M$ predicted below $T_{m}$ which should be observable. For full coverage, the solid line shows the results for all three Cases. Case II is coverage independent. Figure 3(c) demonstrates that, for Case III, the drop-off in $\mathrm{M}$ occurs at higher $\mathrm{T}$ as the coverage is decreased. Case I results in similar behaviour, but has an even stronger dependence on coverage (not depicted).

Because of the large uncertainties in calculations of the difference of C-down and O-down binding energies, we also considered [see Fig. 3(e)] the limiting case where the binding energy is equal for both vertical orientations of the dipole (i.e., $\mathrm{H} \rightarrow 0$ ). Here, the low temperature configuration is ferroelectric with most molecules adsorbed O-down, as this minimizes $U_{\text {dipole. }}$ Observable orientation changes occur at $T<T_{m}$. Note the substantial decrease in $\mathrm{T}$ for a given $\mathrm{M}$ with decreasing coverage. This suggests a coverage dependent disordered-to-ordered ferroelectric phase change may be observable if the difference in binding energy for vertical orientations is small or vanishing. The transition from one ferroelectric state favored by adsorbate-substrate interactions (e.g., mostly C-down) to the other ferroelectric state favored by the adsorbate-adsorbate interactions (e.g., mostly O-down) occurs when the argument of the tanh function in Eq. 4(c) equals zero at $\mathrm{M}=0$, that is when $\mathrm{H}=-\mathrm{Lz} \Theta$. At full coverage, this occurs at $\mathrm{H}=2.76 \mathrm{meV}$. In Fig. 3(c), with $\mathrm{H}=2.0 \mathrm{meV}$, a transition occurs from mostly O-down FE to mostly C-down FE with decreasing coverage. It must be emphasized that most theoretical and experimental evidence for $\mathrm{CO}$ on MgO favors high $\mathrm{H}$ solutions with only a single C-down FE phase. However, our extensions to lower values of $\mathrm{H}$ provide insight into other aspects of our spin-lattice model and predictions for modified CO adsorption and other adsorbed dipolar systems as discussed below.

\section{DISCUSSION}

\section{A. Comparison with Experiment}

We estimate that changes of $\lesssim 20 \%$ in $|\mathrm{M}|$ should be experimentally observable. Pacchioni et al. ${ }^{22}$ have calculated ir spectral shifts separated by $52 \mathrm{~cm}^{-1}(6.5 \mathrm{meV})$ for the two vertical orientations of CO adsorbed on MgO; 
Dovesi et al. ${ }^{35}$ estimate this separation to be $\sim 20 \mathrm{~cm}^{-1}$. Given such large separations one expects small changes in $\mathrm{M}$ would be observable with standard ir spectroscopy, although intrinsic peak broadening may limit observation of changes in dipole ordering and other effects, such as molecular tilting, may also lead to such spectral shifts. Further, dipole coupling interaction may affect the relative ir peak intensities or even mask the entire signal near $|\mathrm{M}| \approx 0.5{ }^{45}$ Photoemission spectroscopy of adsorbed $\mathrm{C}$ or $\mathrm{O}$ core levels may provide an alternate method to monitor M; these measurements may be difficult due to the high energy resolution required. Second harmonic generation and sum/difference spectroscopy possess high resolution and surface sensitivity which should make them ideal techniques to study these predictions of variations in $\mathrm{M}^{46}$ The contrast of $\mathrm{C}$ and $\mathrm{O}$ atoms for $\mathrm{x}$-ray, electron or neutron diffraction is not sufficient to readily distinguish the two vertical orientations of $\mathrm{CO} .{ }^{9}$ Helium atom scattering may be able to determine CO orientation, although measurement would be difficult and require extensive interpretation. ${ }^{34,43}$

There is very limited direct information on the dipole structure or phase transitions of $\mathrm{CO}$ on $\mathrm{MgO}$ or $\mathrm{NaCl}$, particularly at lower coverages, which can be compared with our theoretical predictions. Our results suggest the CO-substrate interactions will primarily determine the orientation of the admolecules. The observed ir spectral blue shift $^{44}$ for CO on MgO at higher T and lower coverage is consistent with the spectral shift calculated for the C-down orientation. $^{22,42}$ This would corroborate the theoretical models which predict a stronger binding energy for the C-down orientation as the driving mechanism of the thermodynamics of submonolayer CO on MgO.

Recent studies of $\mathrm{CO}$ on $\mathrm{NaCl}$ using He diffraction, ${ }^{16}$ at a coverage of $\Theta=1 \mathrm{ML}$, for $30 \mathrm{~K}<\mathrm{T}<53 \mathrm{~K}$, found the $\mathrm{CO}$ structure is commensurate with the $\mathrm{NaCl}(100)$ surface. At $\mathrm{T}<30 \mathrm{~K}$, observed doubling of the unit cell is consistent with alternately tilted $\mathrm{CO}$ molecules. This tilting, has also been observed in ir polarization studies. ${ }^{29,30}$ The diffraction and ir studies suggest that at a coverage of $\Theta=1 \mathrm{ML}$ for $\mathrm{CO}$ on $\mathrm{NaCl}$ and $\Theta=0.5 \mathrm{ML}$ for $\mathrm{CO}$ on $\mathrm{MgO}$, the quadrupole interactions may influence the orientation of the $\mathrm{CO}$ molecules. Lower coverage structural studies of these two systems seem warranted, to determine first if low-density square, commensurate superlattices exist, and to determine at what coverages the quadrupole interactions begin to affect the orientation of the $\mathrm{CO}$ admolecules.

\section{B. Other Physisorbed Dipolar Systems and Phase Transitions}

Our simple spin model could be applied to other physical systems to test its generality and to look for different phases and transitions. Alternative phase transitions, including those discussed above as $\mathrm{H} \rightarrow 0$, may occur or existing phase transitions may be shifted to experimentally observable temperatures as the relative strengths of the substrate-adsorbate ( $\mathrm{H}$ in Eq. 4) and adsorbate-adsorbate interactions ( $\mathrm{J}$ and $\mathrm{L}$ ) are varied. For example, disordered-to-ordered ferroelectric or antiferroelectric transitions could be moved to lower temperatures by decreasing the ratio $\mathrm{H} / \mathrm{zJ}$ (see Eq. 4). Coverage driven transitions from one ferroelectric state to the other may also become observable as this ratio is decreased. The ratio could be modified by choosing systems with increased interaction energies. Alternately, the substrate electric field could be reduced (lowering $\mathrm{p}_{\text {ind }}$ ) by choosing a different substrate, by investigating the second or higher adsorbed layer, or by studying substrates plated with a relatively inert buffer layer such as $\mathrm{Ar}$ or Xe. If a monolayer of $\mathrm{Xe}$ is used to pre-plate $\mathrm{MgO}$ or $\mathrm{NaCl}$, a $30 \%$ reduction in the substrate interaction energy would occur (assuming the binding energy falls off exponentially). ${ }^{25}$ This would mitigate dipole induction effects significantly, and reduce the binding energies for CO adsorption.

An antiferroelectric state in a physisorbed system requires $\mathrm{p}_{\text {ind }}<\mathrm{p}_{\text {nat }}$ so that the two vertical orientations have opposed net dipoles, and alternating alignment is energetically favored. Two ways to realize such a system would be to adsorb molecules with a smaller polarizability, and hence a smaller induced dipole moment, or to adsorb molecules with a larger natural dipole moment. For instance, $\mathrm{CX}_{3} \mathrm{Y}$ dipolar molecules (as discussed below) could be adsorbed to an ionic crystal. ${ }^{13}$

Indeed, in several other systems, electric dipole ordering is observed. Of particular interest are halogenated methanes (molecules of the form $\mathrm{CX}_{n} \mathrm{Y}_{4-\mathrm{n}}$ with $\mathrm{n}=1,2,3$ and $\mathrm{X}, \mathrm{Y}=\{\mathrm{H}, \mathrm{F}, \mathrm{Cl}, \mathrm{Br}, \mathrm{I}\}$ ) which exhibit large natural dipole moments and small polarizabilities compared to CO. ${ }^{47}$ The adsorption of these molecules on graphite has been studied extensively. Antiferroelectrically ordered phases have been observed for $\mathrm{CF}_{3} \mathrm{H}^{48}$ and for $\mathrm{CH}_{3} \mathrm{Cl}$ and $\mathrm{CH}_{3} \mathrm{Br}^{47}$ Other electrically ordered phases have been observed for $\mathrm{CF}_{3} \mathrm{Cl}^{49}$ For graphite coated with $\mathrm{Xe}^{4} \mathrm{CH}_{3} \mathrm{~F}$ is observed to have a ferroelectric phase not seen on bare graphite. ${ }^{47}$ However, none of these systems have cubic symmetry and do not lend themselves easily to application of our simple model. In principle, such dipolar adsorption on graphite could be modeled by an Ising model on a triangular lattice.

\section{ACKNOWLEDGMENTS}

The authors would like to thank F.Y. Wu and C.Y. Pan for help in formulating the spin-lattice problem, S. Fain, H. Taub, and G. Scoles for discussion on the application to physisorbed systems, and M. Riffe, C. Torre, and J. Wheeler for useful comments. This work has been supported by the Donors to the Petroleum Research Fund. 


\section{REFERENCES}

[1] See for example, W. Steele, Chem. Rev. 93 (1993) 2355 and Adsorption on Ordered Surfaces of Ionic Solids and Thin Films, edited by E. Umback and H.-J. Freund (Springer-Verlag, Berlin, 1993).

[2] See for example R.K. Pathria, Statistical Mechanics, (Pergamon Press, New York, 1972).

[3] F.Y. Wu, private communication.

[4] J. Sivardière and J. Lajzerowicz, Phys. Rev. A 11 (1975) 2090.

[5] F.Y. Wu, Chinese J. Phys. 16 (1978) 153.

[6] W. Hoston and A. N. Berker, Phys. Rev. Lett. 67 (1991) 1027; W. Hoston, MIT, MS Thesis, unpublished (1991).

[7] M. Blume, V.J. Emery and R.B. Griffiths, Phys. Rev. A 4 (1971) 1071.

[8] J.M. Yeomans, Statistical Mechanics of Phase Transitions, (Oxford University Press, Oxford, 1992), p. 41.

[9] T.E. Burns, Utah State Univ., Ph.D. Thesis, unpublished (1994).

[10] M. Schick and W.-H. Shih, Phys. Rev. B 34 (1986) 1797.

[11] J. Lajzerowicz and J. Sivardiere, Phys. Rev. A 11 (1975) 2079.

[12] C.N. Yang and T.D. Lee, Phys. Rev. 87 (1952) 410.

[13] T. Burns and J.R. Dennison, Bull. Am. Phys. Soc. 40 (1995) 128; (unpublished).

[14] M. Blume and R.E. Watson, J. Appl. Phys. 38 (1967) 991.

[15] Summations for $U_{\text {dipole }}$ and $U_{\text {quad }}$ included the first 100 nearest neighbor shells (828 admolecules) explicitly, with an effective medium integral correction $(\ll 0.01 \%)$ at larger radii, where the discrete nature of the lattice becomes negligible. We estimate the error in $\mathrm{U}_{\text {dipole }}$ to be $\leq 1 \%$ and the error in $\mathrm{U}_{\text {quad }}$ to be $« 1 \%$. For $\mathrm{CO}$ on $\mathrm{NaCl}$ and $\mathrm{MgO}$, truncation of $U_{\text {disp }}$ to first nearest neighbors introduces $\lesssim 0.1 \%$ error. See Ref. 9 .

[16] A. Ben-Ephraim et al., J. Chem. Phys. 89 (1988) 3840.

[17] Spin projection operators $\left[\mathbf{P}_{\mathrm{i}}^{\mathrm{u}}=1 / 2\left(\mathrm{~S}_{\mathrm{i}}^{2}+\mathrm{S}_{\mathrm{i}}\right), \mathbf{P}_{\mathrm{i}}^{0} \equiv\left(1-\mathrm{S}_{\mathrm{i}}^{2}\right), \mathbf{P}_{\mathrm{i}}^{\mathrm{d}}=1 / 2\left(\mathrm{~S}_{\mathrm{i}}^{2}-\mathrm{S}_{\mathrm{i}}\right)\right]$ were first used to write the dipole

$$
U_{\mathrm{dipok}}^{i}=\frac{1}{4 \pi \epsilon_{0}} \sum_{i \neq j} \frac{p_{i} p_{j} \boldsymbol{P}_{i} \boldsymbol{P}_{j}}{r_{i j}^{3}},
$$

interaction as and then like terms of $\mathrm{S}$ and $\mathrm{S}^{2}$ were collected. Refer to Ref. 4.

[18] Full coverage refers to the densest allowed commensurate, square superlattice. For $\mathrm{CO}$ on $\mathrm{NaCl}$, full coverage is at $\Theta=1 \mathrm{ML}$. For $\mathrm{CO}$ on $\mathrm{MgO}$, full coverage is at $\Theta=1 / 2 \mathrm{ML}$. $1 \mathrm{ML}$ is defined as a coverage of one admolecule for each cation site.

[19] W. Brakeman and A.L. Mossman, Matheson Gas Data Book, (Matheson, Lyndhurst, 1976), pp. 130-137, 522-530.

[20] D.E. Storgyn and A.P. Storgyn, Mol. Phys. 11 (1966) 371.

[21] J.E. Gready, G.B. Backsay and N.S. Hush, Chem. Phys. 31 (1978) 375.

[22] G. Pacchioni, G. Cogliandro and P.S. Bagus, Int. J. of Quant. Chem. 42 (1992) 1115.

[23] H.H. Richardson and G.E. Ewing, J. Elec. Spectosc. Rel. Phenom. 45 (1987) 99.

[24] D. Schmicker et al., J. Chem. Phys. 95 (1991) 9412.

[25] R. Gevirzman, Y. Kozirovski, and M. Folman, Trans. Fara. Soc. 65 (1968) 2206.

[26] H.H. Richardson, C. Baumann, and G.E. Ewing, Surf. Sci. 185 (1987) 15.

[27] H.-C. Chang, H.H. Richardson, and G.E. Ewing, J. Chem. Phys. 89 (1988) 7561.

[28] J. Heidberg et al., J. Elec. Spectrosc. Relat. Phenom. 45 (1987) 87; 54/55 (1990) 945.

[29] J. Heidberg et al. in Adsorption on Ordered Surfaces of Ionic Solids and Thin Films, edited by E. Umback and H.-J. Freund (Springer-Verlag, Berlin, 1993), pp. 46-55.

[30] J. Heidberg et al., J.Chem. Phys. 95 (1991) 9408; Surf. Sci. 269/270 (1992) 128.

[31] H.H. Richardson and G.E. Ewing, J. Phys. Chem. 91 (1987) 5833.

[32] A. Lakhilfi, Mol. Phys. 78 (1993) 659.

[33] S. Picaud et al., Surf. Sci. 294 (1993) 149.

[34] M.-N. Carre' et al., Surf. Sci. 347 (1996) 128.

[35] R. Dovesi et al., Surf. Sci. 186 (1987) 267.

[36] E.A. Colburn and W.C. Mackrodt, Surf. Sci. 117 (1982) 571; 143 (1984) 391.

[37] A. Lakhlifi and C. Girardet, Surf. Sci. 241 (1990) 400.

[38] C. Minot, M.A. Van Hove and J.-P. Biberian, Surf. Sci. 346 (1996) 283.

[39] E. Escalona-Platero et al., Faraday Disc. Chem. Soc. 80 (1985) 183.

[40] S.A. Pope et al., Surf. Sci. 139 (1984) 299.

[41] P. Audibert, M. Sidoumou and J. Suzanne, Surf. Sci. 273 (1992) L467-L471; V. Panella et al., J. Phys. (Paris) 4 (1994) 905.

[42] J. Heidgerg et al., Surf. Sci. 331-333 (1995) 1467.

[43] R. Gerlach et al., Surf. Sci. 331-333 (1995) 1490. 
[44] Jian-Wei He et al., Surf. Sci. 261 (1992) 164.

[45] P. Hollins and J. Pritchard, Prog. Surf. Sc. 19 (1985) 275; B.N. Persson and R. Ryberg, Phys. Rev. B 24 (1981) 6954.

[46] Y.R. Shen, Surf. Sci. 299/300 (1994) 551; Nature 337(9) (1989) 519.

[47] P.A. Rowntree, G. Scoles, and J.C. Ruiz-Suarez, J. Phys. Chem. 94 (1990) 8511.

[48] K. Knorr and W. Wiechert, Phys. Rev. B 37 (1987) 3524.

[49] W. Weimer, K. Knorr, and H. Wiechert, Z. Phys. B - Cond. Mat. 73 (1988) 235. 
Table 1: Natural and induced dipole moments ${ }^{\mathrm{a}}$ of $\mathrm{CO}$ as a function of orientation.

\begin{tabular}{||c||c|c|c|c||}
\hline \hline \multirow{2}{*}{\begin{tabular}{l} 
Adsorbate \\
\multirow{2}{*}{ Orientation }
\end{tabular}} & \multicolumn{2}{c|}{ CO on NaCl (100) } & \multicolumn{2}{c||}{ CO on MgO (100) } \\
\cline { 2 - 5 } & Natural & Induced & Natural & Induced \\
\hline \hline $\mathrm{p}_{\mathrm{u}} \rightarrow \mathrm{p}_{\mathrm{c}}(\mathrm{C}-$ down) & $+0.112 \mathrm{D}^{\mathrm{b}}$ & $+0.292 \mathrm{D}^{\mathrm{c}}$ & $+0.112 \mathrm{D}^{\mathrm{b}}$ & $+0.494 \mathrm{D}^{\mathrm{d}}$ \\
\hline $\mathrm{p}_{\mathrm{d} \rightarrow \mathrm{p}_{\mathrm{o}}(\mathrm{O}-\text { down })}$ & $-0.112 \mathrm{D}^{\mathrm{b}}$ & $+0.287 \mathrm{D}^{\mathrm{c}}$ & $-0.112 \mathrm{D}^{\mathrm{b}}$ & $+0.574 \mathrm{D}^{\mathrm{d}}$ \\
\hline
\end{tabular}

$1 \mathrm{D}=3.336 \times 10^{-30} \mathrm{C}-\mathrm{m}$

b Ref. 19.

c Ref. 21.

d Ref. 22.

Table 2: Binding energies of $\mathrm{CO}$ as a function of orientation and coverage (1).

\begin{tabular}{|c|c|c|c|c|}
\hline \multirow[t]{2}{*}{ Adsorbate } & \multirow{3}{*}{$\begin{array}{c}\mathrm{CO} \text { on } \mathrm{NaCl} \\
(\mathrm{eV})^{\mathrm{a}}\end{array}$} & \multicolumn{3}{|c|}{ CO on MgO (100) } \\
\hline & & \multicolumn{2}{|c|}{ Lower Limit $^{\mathrm{b}}$} & \multirow{2}{*}{$\begin{array}{r}\text { Upper Limit }^{\mathrm{C}} \\
(\mathrm{eV})\end{array}$} \\
\hline Orientation & & 1 & $(\mathrm{eV})$ & \\
\hline \multirow{2}{*}{, bd (C-down) } & \multirow{2}{*}{0.417} & 0.5 & 0.179 & \multirow{2}{*}{0.390} \\
\hline & & \#0.25 & 0.194 & \\
\hline \multirow{3}{*}{, bu (O-down) } & \multirow{3}{*}{0.171} & 0.5 & 0.143 & \multirow{3}{*}{0.273} \\
\hline & & & & \\
\hline & & \#0.25 & 0.184 & \\
\hline
\end{tabular}

\footnotetext{
Ref. 21.

b Ref. 35.

c Ref. 36b.
} 
Table 3: (a) CO-CO interaction energies per site for $\mathrm{CO}$ on $\mathrm{MgO}$ and $\mathrm{CO}$ on $\mathrm{NaCl}$ at full coverage. ${ }^{0} \mathrm{U}^{+}$dipole, ${ }^{0} \mathrm{U}^{0}$ dipole, and ${ }^{\circ} \mathrm{U}^{-}$dipole are the dipole interaction energies for all O-down ferroelectric, disordered, and all C-down ferroelectric infinite lattices, respectively. $\mathrm{U}^{0}{ }_{\text {quad }}$ is the quadrupole-quadrupole interaction energy for the CO molecules. $\mathrm{U}_{\text {disp }}$ is the dispersion (hard-core repulsion) energy between nearest neighbor CO molecules. (b) The spin lattice model Hamiltonian interaction energy parameters $\mathrm{J}_{0}, \mathrm{~K}_{0}, \mathrm{~L}_{0}$, $\mathrm{H}_{0}$, and ) ${ }_{0}$ as defined in Eq. (2), at full coverage and low temperature.

(a)

\begin{tabular}{||c||c|c|c|c|c||}
\hline \hline System & $\begin{array}{c}{ }^{0} \mathrm{U}^{+}{ }_{\text {dipole }} \\
(\mathrm{meV})\end{array}$ & $\begin{array}{l}{ }^{0} \mathrm{U}_{\text {dipole }}^{0} \\
(\mathrm{meV})\end{array}$ & $\begin{array}{c}{ }^{0} \mathrm{U}^{-}{ }_{\text {dipole }} \\
(\mathrm{meV})\end{array}$ & $\begin{array}{c}\mathrm{U}_{\text {quad }}^{0} \\
(\mathrm{meV})\end{array}$ & $\begin{array}{c}\mathrm{U}_{\text {disp }}^{0} \\
(\mathrm{meV})\end{array}$ \\
\hline \hline $\mathrm{CO} / \mathrm{MgO} ; 1=1 / 2 \mathrm{ML}$ & 15.4 & 20.6 & 26.5 & 15.0 & 6.99 \\
\hline $\mathrm{CO} / \mathrm{NaCl} ; 1=1 \mathrm{ML}$ & 2.75 & 6.81 & 13.5 & 19.7 & 18.2 \\
\hline
\end{tabular}

(b)

\begin{tabular}{||c||l|l|l|l|l||}
\hline \hline System & $\mathrm{J}_{\mathrm{o}}(\mathrm{meV})$ & $\mathrm{K}_{\mathrm{o}}(\mathrm{meV})$ & $\mathrm{L}_{\mathrm{o}}(\mathrm{meV})$ & $\mathrm{H}_{\mathrm{o}}(\mathrm{meV})$ & ) $_{\mathrm{o}}(\mathrm{meV})$ \\
\hline \hline $\mathrm{CO} / \mathrm{MgO} ; 1=1 / 2 \mathrm{ML}$ & -0.12 & -10.6 & -0.69 & +58.5 & +332 \\
\cline { 4 - 6 } & & & & +5.21 & +189 \\
\hline $\mathrm{CO} / \mathrm{NaCl} ; 1=1 \mathrm{ML}$ & -0.33 & -11.2 & -0.67 & +123 & +294 \\
\hline
\end{tabular}


(a)

(b)
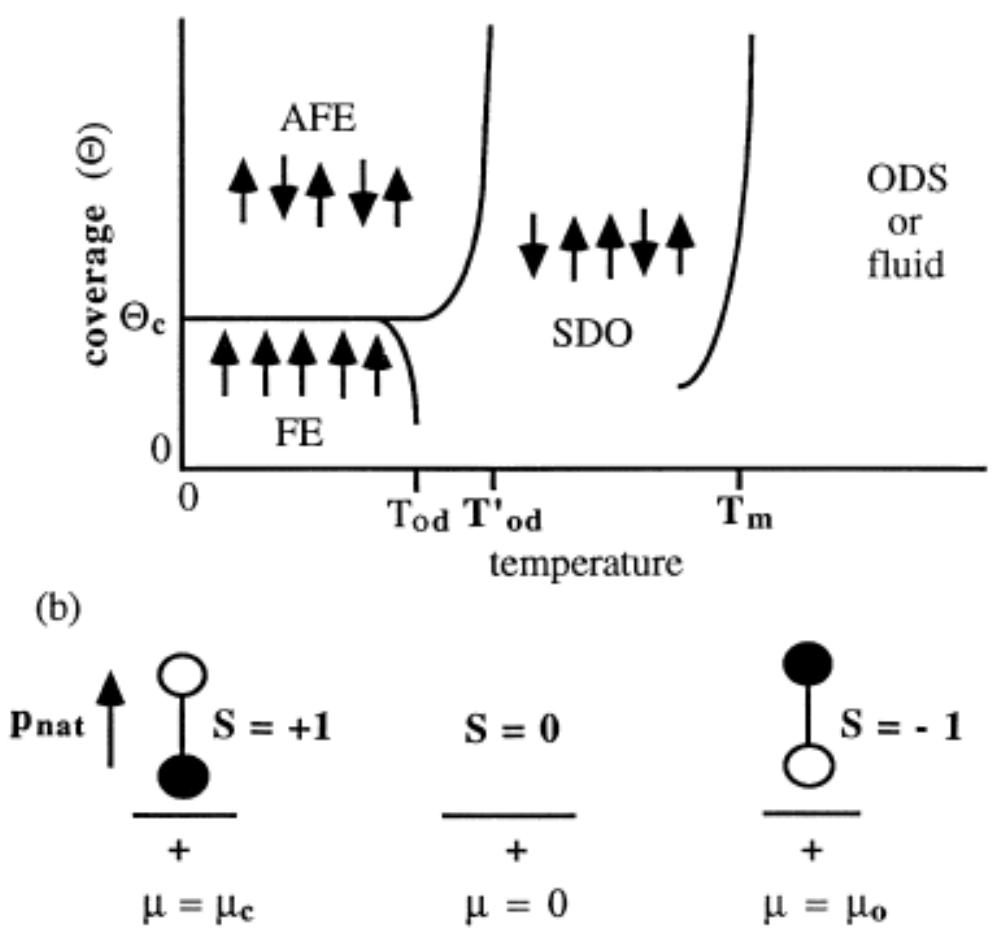

(c)

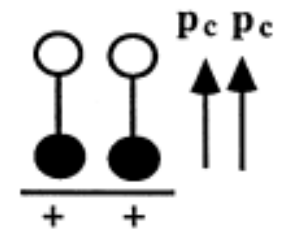

Jec

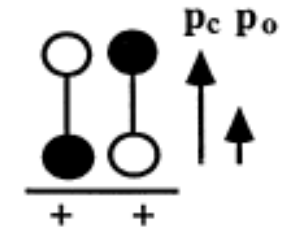

$\mathbf{J}_{\text {co }}$

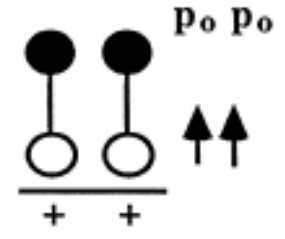

Joo

i)

iii)

Fig. 1. (a) Schematic diagram of the possible phase transitions for a hypothetical lattice of perpendicular electric dipoles interacting with an external field. AFE represents an antiferroelectric or ferrielectric phase, FE is a ferroelectric phase, SDO is a spin-disordered phase, and ODS is an orientationally disordered solid or fluid phase. The temperatures $\mathrm{T}_{\text {od }}, \mathrm{T}_{\text {od }}^{\prime}$, and $\mathrm{T}_{\mathrm{m}}$ represent the two dipole-orientation order-disordered transitions and the translational orientation order-disorder (melting) transitions, respectively. (b) Spin assignment for $\mathrm{CO}$ adsorbed vertically above a cation lattice site $(+)$ on an ionic crystal surface. The circles represent $\mathrm{C}(\bullet)$ and $\mathrm{O}(\circ)$ atoms. (c) Dipole and molecular orientations for the three configurations of $\mathrm{CO}$ adsorbed vertically above metal ion lattice sites on ionic crystal surfaces. 


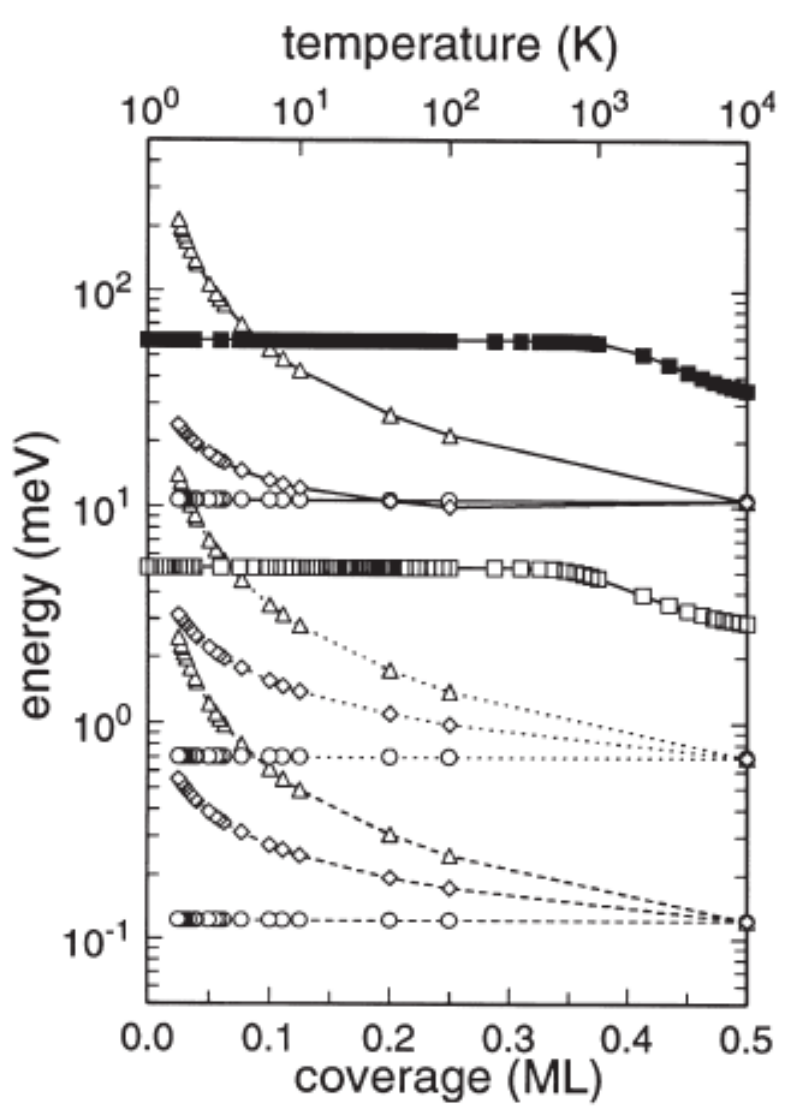

(a)

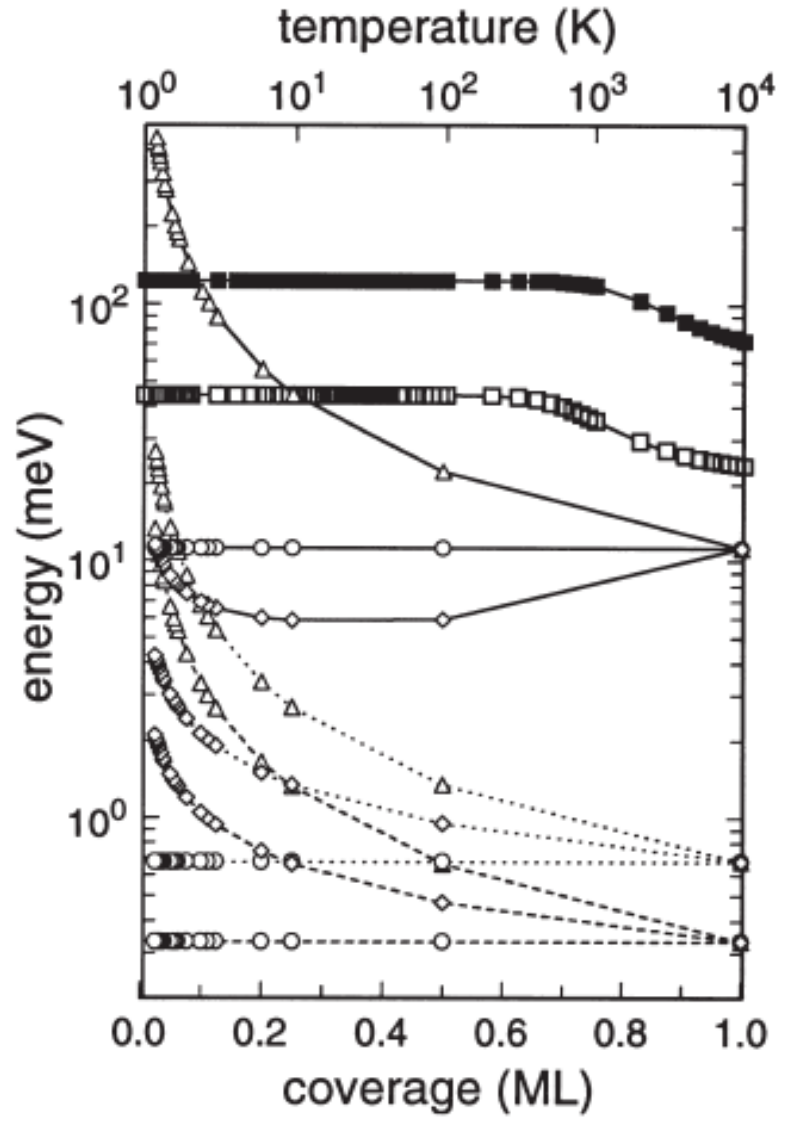

(b)

Fig. 2. The spin lattice model Hamiltonian interaction energy parameters for (a) CO on $\mathrm{MgO}$ and (b) CO on NaCl. Figures show -J (dashed lines), -K (solid lines)) and -L (dotted lines) as functions of coverage. The symbols indicate the strongly attractive (Case I; F, weak (Case II; è), and strongly repulsive (Case III; $\AA$ ) interaction limits, respectively. $\mathrm{H}(\sim$-- lower limit and $\ddot{A}$-- upper limit) at full coverage is shown as a function of temperature. The symbols indicate the specific temperatures and superlattice coverages considered in this study. 


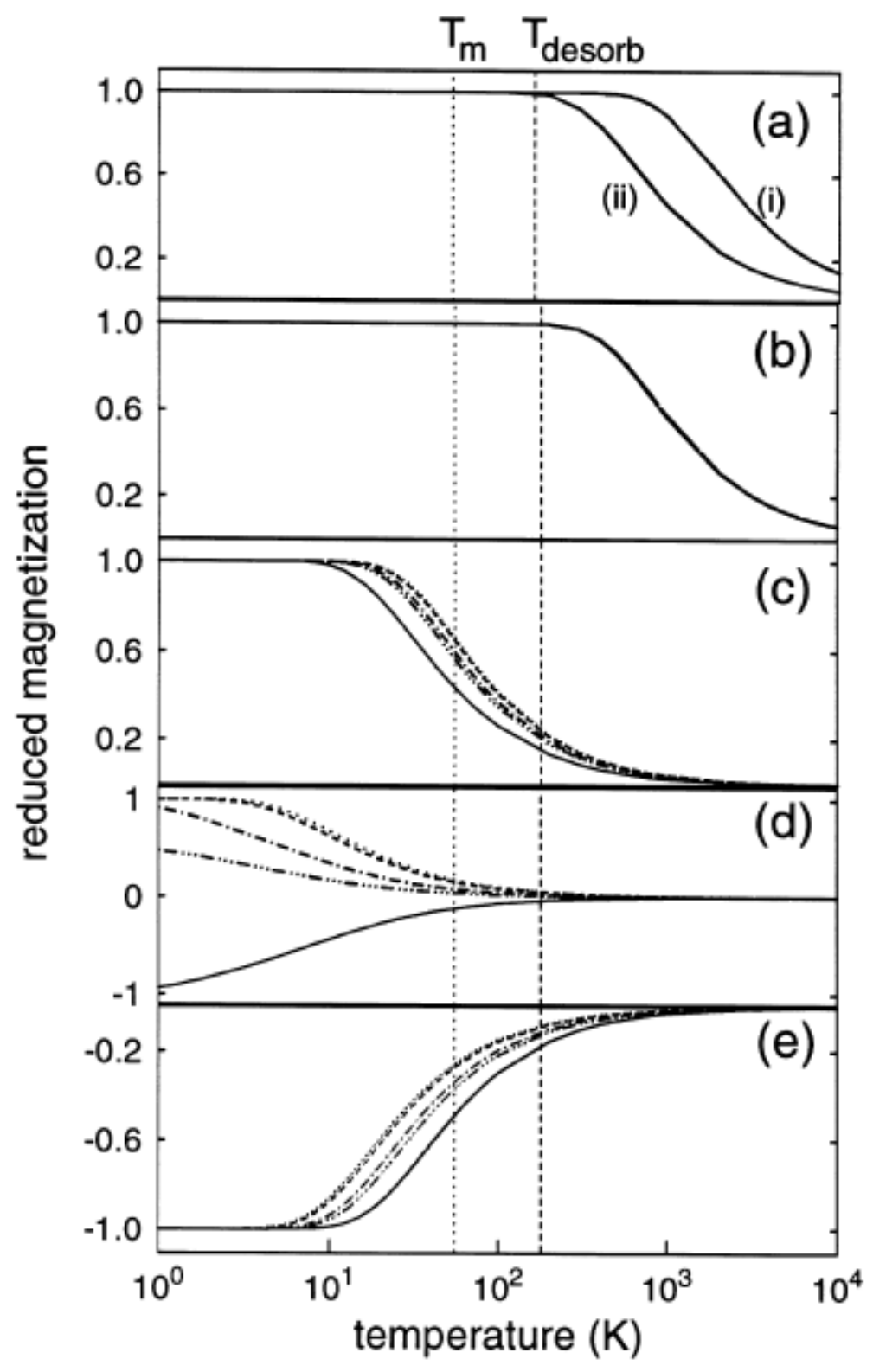

Fig. 3. Reduced magnetization (M/1) as a function of coverage. (a) $\mathrm{CO}$ on $\mathrm{NaCl}$ with $\mathrm{H}_{0}=123 \mathrm{meV}$. $\mathrm{CO}$ on $\mathrm{MgO}$ with (b) $\mathrm{H}=58.6 \mathrm{meV}$, (c ) $\mathrm{H}=5.2 \mathrm{meV}$, (d) $\mathrm{H}=2.0 \mathrm{meV}$ and (e) $\mathrm{H}=0.0 \mathrm{meV}$. Curves in (c-e) indicate coverages of $1=1 / 2 \quad \mathrm{ML}$ (solid), $1=1 / 4 \mathrm{ML}$ (dot-dot-dot-dashed), $1=1 / 5 \mathrm{ML}$ (dot-dashed), 1=1/8 ML (dot-dash) and 1=1/9 (dotted), respectively, for the strongly repulsive (Case III) limit. The solid curves are also valid for Case II at all coverages and Case I at full coverage. Note the logarithmic temperature scale. 